\title{
Incointri
}

Anno 35, 2020 / Fascicolo 1 / p. 146-148 - www.rivista-incontri.nl - http://doi.org/10.18352/incontri.10343

(c) The author(s) - Content is licensed under a Creative Commons Attribution 3.0 Unported License - Publisher: Werkgroep Italië Studies, supported by Utrecht University Library Open Access Journals

\section{Un oggetto delicato, da maneggiare con grande cura Tradurre e ritradurre i classici}

\author{
Recensione di: Stefania Ricciardi (a cura di), Tradurre e ritradurre $i$ \\ classici. 'Testo a fronte' 60, Milano, Marcos y Marcos, 2019, 263 p., \\ ISBN: 8871689593, € 25,00.
}

\section{Linda Pennings}

'Perché leggere i classici'? Il discorso che Italo Calvino formula nel suo famoso saggio del 1981, per cercare una risposta a questa domanda, potrebbe applicarsi, mutatis mutandis, anche alla traduzione dei classici. Tant'è vero che verso la fine del saggio lo scrittore si accorge di aver citato, oltre alle varie opere straniere, solo Leopardi come nome della letteratura italiana. Calvino afferma che sono proprio i classici di tutte le culture a farci capire 'chi siamo e dove siamo arrivati',' tramite un confronto con l'Altro, proveniente da diversi tempi e luoghi. Sembra lecito affermare allora che l'esortazione calviniana alla lettura dei classici sia rivolta anche alla lettura dei classici tradotti, e dunque alla loro traduzione.

Perché tradurre, o anzi, ritradurre i classici? Secondo la prima delle quattordici definizioni proposte da Calvino, un classico è un'opera che piuttosto di essere letta è continuamente riletta; così la ritraduzione è una pratica che in genere si riserva proprio alle opere alle quali si tende ad assegnare il predicato di classico, antico o moderno. Il tema si trova affrontato nella sezione tematica della rivista 'Testo a fronte' 60 (primo semestre 2019), intitolata appunto Tradurre e ritradurre i classici e volta a indagare, nelle parole della curatrice Stefania Ricciardi, 'alcune questioni cruciali: come si affronta un classico, come si "svecchia" una traduzione che appare datata, come si arriva a tradurre in una lingua contemporanea che dia l'illusione acustica del vintage, che renda cioè il tono e il timbro dell'antico con parole moderne, e che colga quanto sussiste di moderno in quel tono e quel timbro antichi' (p. 5).

Sono solo alcune delle questioni discusse durante la giornata di studi svoltasi presso l'Istituto Italiano di Cultura di Bruxelles, il 7 novembre 2018. Negli otto contributi le considerazioni relative alle problematiche della ritraduzione - di llide Carmignani su Cent'anni di solitudine di Gabriel García Márquez, di Franca Cavagnoli su Il grande Gatsby

\footnotetext{
${ }^{1}$ Italo Calvino, 'Perché leggere i classici' (1981), in Saggi 1945-1985, a.c.d. M. Barenghi, Mondadori, Milano 1995, vol. II, p. 1824.
} 
di Francis Scott Fitzgerald, di Fabio Pedone su Finnegans Wake di James Joyce e di Stefania Ricciardi su Moneta del sogno di Marguerite Yourcenar - si alternano e si intrecciano a quelle in merito alle peculiarità del tradurre i classici, di Alessandro Niero su Noi di Evgenij Zamjatin e di Franco Paris sulla lirica Visio Tondalis di Hugo Claus, come pure alle riflessioni sulla traduzione tout court presenti in tutti i contributi e negli estratti dei due saggi pubblicati in volume che aprono e chiudono la sezione, rispettivamente di Mariarosa Bricchi (Tradurre, e la grammatica) e di Enrico Terrinoni (Tradurre noi stessi).

Che si tratti di riflessioni, cioè di pensieri scaturiti direttamente dall'esperienza, e non (o poco) di teoria traduttologica, è messo in particolare rilievo nell'introduzione al volume, dove la curatrice richiama le parole di Antoine Berman per sostituire ai concetti usuali di teoria e pratica quelli di expérience e réflexion. Se effettivamente una netta dicotomia tra teoria e pratica in campo di traduzione al giorno d'oggi appare insostenibile, è anche evidente che il rifiuto della 'teoria' va letto come un distanziamento dalla scienza normativa e dogmatica della traduzione, formatasi sulla scia delle tendenze scientifiche negli studi letterari e linguistici, a cui però negli ultimi decenni sono seguite tendenze radicalmente diverse, orientate sulla poetica e sull'ermeneutica, sulla soggettività del traduttore e sulla pluralità della traduzione.

In effetti, come afferma Ricciardi, 'la traduzione non può rinunciare al pensiero e convive efficacemente con quelle problematiche della traduttologia che non veicolano concetti astratti ma implicano l'esperienza' (p. 8). E così nei contributi non mancano i riferimenti a Paul Ricoeur, Antoine Berman, Umberto Eco, Susan Sontag, Franco Buffoni e altri, nel filo conduttore di un concetto di traduzione incentrato sull'imperfezione, la provvisorietà, la trasformazione, la mobilità, la manipolazione, la contingenza e la differenza. Lungi da qualsiasi nozione di assoluto o definitivo, la traduzione si presenta piuttosto, a dirla con Ricciardi, come 'un contributo critico all'interpretazione del testo' (p. 5).

La traduzione concepita come un'interpretazione necessariamente parziale, una 'lettura' condizionata dalle esigenze del contesto culturale e dalle esperienze soggettive del traduttore, spiega e legittima per la sua stessa natura il fenomeno della ritraduzione, cioè della pubblicazione di più traduzioni di un'opera nella medesima lingua, in genere separate da un periodo di tempo più o meno lungo. Però il lettore che si aspetti di trovare discussi il concetto e i motivi della ritraduzione resterà forse in un primo momento deluso, in quanto, oltre al riferimento alla visione famosa e controversa di Berman - secondo cui ogni ritraduzione si avvicinerebbe di più all'essenza vera e alla voce autentica dell'opera originale, in un processo di compimento della traduzione ideale -, non sono menzionati studi più recenti in cui tale visione è confutata, modificata o integrata con numerose altre prospettive, fondate su ricerche concrete e specifiche. ${ }^{2}$

Tuttavia, ciò è in linea con l'approccio scelto, ovvero quello di partire dall'esperienza e dalla riflessione dei traduttori. Le singole trattazioni delle opere ritradotte infatti fanno emergere la complessità dei motivi che possono indurre alla realizzazione di una nuova traduzione: l'invecchiamento della lingua o della strategia traduttiva di una traduzione esistente, i cambiamenti ideologico-culturali del contesto di arrivo o della posizione culturale dell'autore tradotto, la disponibilità di nuove edizioni

\footnotetext{
${ }^{2}$ Antoine Berman, 'La retraduction comme espace de traduction', Palimpsestes 4 (1990), pp. 1-7. Cfr. per es. Annie Brisset, 'Retraduire ou le corps changeant de la connaissance. Sur l'historicité de la traduction', Palimpsestes 15 (2004), pp. 39-67, Lawrence Venuti, 'Retranslations: The Creation of Value', Bucknell Review 47, 1 (2004), pp. 25-38, Sharon Deane-Cox, Retranslation, Translation, Literature and Reinterpretation, New York, Bloomsbury, 2014.
} 
del testo originale, la nascita di una collana dedicata alla riscoperta dei classici o l'allestimento di un'edizione dotata di un apparato paratestuale, la necessità di emendare errori, nonché la sfida accolta dal traduttore di elaborare una nuova interpretazione o una diversa resa stilistica.

Oltre al perché ritradurre, è soprattutto il come tradurre i classici a dare lievito ai discorsi dei traduttori. Al di là della diversità delle problematiche, strategie e soluzioni discusse, l'idea che regna sovrana è che, come affermato da Franca Cavagnoli, 'un classico è un oggetto delicato, da maneggiare con grande cura': ${ }^{3}$ si parla di responsabilità e di rispetto, anzi di devozione, nel ricreare la voce della scrittura nella sintassi e nella punteggiatura, nel ritmo e nei valori fonici, nei culturemi e nella diversità, nei neologismi e nell'intertestualità. Delle ammirevoli interpretazioni e ricreazioni danno prova i brani delle traduzioni pubblicate raccolti in appendice, che - sia detto per inciso - il lettore esigente avrebbe desiderato leggere con il testo a fronte.

'Un classico è un libro che non ha mai finito di dire quel che ha da dire.' (p. 1818) La sesta definizione proposta da Calvino ha sempre affascinato ma non del tutto convinto Enrico Terrinoni, il quale osserva al riguardo che ' $\mathrm{i}$ testi, in quanto lettera che muore, non hanno da dire alcunché finché non li facciamo risorgere leggendoli' (p. 87); e diciamo pure, traducendoli e ritraducendoli. Dell'infinita plurileggibilità dei classici letterari testimoniano, con ricchezza di spunti ed esempi stimolanti, le esperienze e le riflessioni dei traduttori raccolte in questa lodevole sezione tematica di 'Testo a fronte'.

\author{
Linda Pennings \\ Italië Studies \\ Universiteit van Amsterdam \\ Postbus 1641 \\ 1000 BP Amsterdam (Paesi Bassi) \\ linda.pennings@uva.nl
}

\footnotetext{
${ }^{3}$ La citazione, riferita nell'introduzione (p. 5), è tratta da Franca Cavagnoli, La voce del testo. L'arte e il mestiere di tradurre, Milano, Feltrinelli, 2019 [2012], p. 154.
} 